\title{
Comprehensive evaluation application analysis of the safety of distribution live work on $10 \mathrm{kV}$
}

\author{
Zhu Ming, Jiao Taiming, Gong Hailin \\ Benxi Power Supply Company of National Grid Liaoning Electric Power Co., Ltd. \\ bxwl_650928@126.com
}

Keywords: distribution live work on $10 \mathrm{kV}$; analytic hierarchy process; safety evaluation; fuzzy comprehensive evaluation; safety management; safety level

\begin{abstract}
In this paper, for the safety risks such as complex working environment of the distribution live work on $10 \mathrm{kV}$, the safety risk of the distribution live work on $10 \mathrm{kV}$ of multi-circuits on the same tower in a certain town of Benxi is evaluated by using analytic hierarchy process and fuzzy comprehensive evaluation and the safety evaluation index system of live work including working environment, personnel quality, working equipment and protection, comprehensive management of safe work, field management is set up. Based on the safety evaluation index system of live line work, the relevant quantitative analysis is done, the risk factors of live line work are analyzed, and the safety level of the live work is determined. According to the computation results of safety evaluation of live line work, this method provides a scientific basis for the safety management of distribution live work on $10 \mathrm{kV}$ when live line work of multi-circuits on the same tower is in a relatively good condition.
\end{abstract}

\section{Introduction}

Nowadays, the distribution network live work has been popularized to the county (city) power supply companies, but in order to implement the commitment service to the users, some companies reduce the power outage time and arrange live work as much as possible, while ignoring the safety management which is very dangerous. Many live work teams engage in not only power outage maintenance, and operation, but also live work, so they are easy to bring into the work habit during power outage into live work, resulting in accidents of incorrect or wrong operations in live work. And it is impossible for the power supply companies to do live work every day, and they may have the work task after a long time of live work, and live work personnel engage in other tasks most of the time. Therefore, the daily training of live work is essential. The content of live work daily training includes: the part of live work of "Electrical Safety Regulations", "Live Work Procedures", live work basic knowledge, rules and regulations, hands-on exercises, and the technical clarification and accident case learning before the complex project, so as to constantly improve their theoretical knowledge and working skills.

\section{Methods of distribution live work}

\section{Classification.}

The "Electrical Safety Regulations" enacted by the state divide live work into three sorts of equal potential work, ground potential work, work intermediate potential work. By direct contact or contact by means of an insulating tool, it is divided into direct and indirect live work. However, the classification of distribution live work must be confirmed in accordance with specific work and technical conditions of live work (the technical conditions of live work refer to the safe distance of sufficient clearance where the current flowing through the body does not exceed $1 \mathrm{~mA}$, and field strength of body surface is less than $2.4 \mathrm{kV} / \mathrm{cm}$ ).

\section{Ground potential method.}

The live work of the ground potential method, which is the largest branch of the live work and the basis of other methods, can be expressed as the grounding body --- the human body --- the insulator--- the charged body. Human body and grounding body are at the equal potential. By using 
insulated tools it is able to contact with the charged body and maintain certain safety clearance with charged body at the same time.

\section{Equal potential method.}

Equal potential method of live work can be expressed as the grounding body --- the insulator --the human body --- the charged body, that is, after being insulated from the grounding body through the insulator, the human body can directly carry out a variety of work on the charged body. Due to different voltage levels and electric field intensities, the human body has to take the use of reliable electric field protective measures (such as using a shield at ultra high voltage power transmission line, and using insulated clothing at $10 \sim 35 \mathrm{kV}$ ), in addition to maintaining a certain distance with the grounding body, while the insulation tool cannot be completely exempted, which still plays a role in limiting current to flow through human body.

\section{Intermediate potential method.}

The intermediate potential method of live work can be expressed as the grounding body --- the insulator --- the human body --- the insulator --- the charged body, that is, the human body takes the use of a shorter insulation tool to work on the charged body through the insulator when being insulated from the ground and maintaining a certain distance with the charged body. In fact, the intermediate potential method is far from a method itself because in terms of potential intensities and contact features, it has a dual nature, and its principle is still the same as the equal potential method and the indirect working method.

\section{Management of live work apparatus}

There are strict requirements of keeping and storage of the live work apparatus and it will affect the service life of the live work apparatus, and even threaten the life safety of the operators if they are not kept or stored as required. Therefore, it needs to establish the warehouse for the apparatus as required and arrange special persons to carry out the management of live work apparatus. Some companies just pay attention to the periodic preventive experiments of the live work apparatus, but do not to daily management, mainly including no special personnel in charge of the management of apparatus, no usage records or inspection records. The ingoing and outgoing of apparatus are managed by operators of live work but due to fatigue of the field work, the operators often fail to do checking, which brings safety risks to live work. However, the safety protection equipment are easy to be scratched or stabbed by all kinds of metal components as being used, so careful inspection of equipment and protective equipment during and after the work should be emphasized, to prevent from accident caused by insulation damage. In addition, being airtight, the insulation clothing makes the human body uncomfortable and sweating a lot, especially in the summer, so it will be immersed in the sweat. Therefore, it should be immediately cleaned with a clean towel after use, so that the insulation effect and the service life of the apparatus will not be affected by sweat pollution and the personal safety is protected.

\section{Training and evaluation of operators}

Different from power outage maintenance work, live work requires strictly on work skills, working procedures and operation methods. Only if live work performs strictly in accordance with the plan and steps formulated in advance can the safety be ensured. If the operators, unfamiliar with the rules, do the work casually at their own will, the opposite will result. For example, when the three phase conductors are arranged vertically in the field, the upper wire is connected but the lower two phase wires are not insulated, or the insulating conductor is taken as an insulator, and then it is very easy to occur the inter-phase short-circuit accident. Also, when an operator is working, he often focuses only on the charged body, but not on the influence of the surrounding non charged body or other charged bodies, which sometimes results in fatal accident. Beside, the distribution live work on $10 \mathrm{kV}$ is a work with high input, high skills and high risk, where the theoretical and technical training for the operators must be strengthened and their professional quality must be improved. Training and evaluation have to be done purposefully and continuously in a planned 
manner, to maintain the relative stability of the operators, and ensure that they engage in this work for a long term. At the same time, the operators must obtain the live work certificate enacted by the national live work training center and cannot be trained in the form of apprenticeship, which is the key to ensure the safety of live work. Each unit is proposed to build a power distribution line in the training base to simulate live work and do skills training so as to strengthen the new knowledge and new methods, and give a full play of the role of the training base.

\section{Work ticket system}

Live work is a special work, and its safety depends not only on the field work ways and work steps, but also on the distribution network work mode, load characteristics, etc. The live work tickets of some companies are only filled in by the directors, and issued by the team leader, without checking and signing by the leader in the work area or the leader in charge, which may cause accident in practice for the safety measures are not sufficient for operators. In addition, after the person in charge reads the work ticket in the field, the operators are not required to sign or repeat. However, the operators may be not clear about or misunderstand the job content, which would cause accident. It is suggested that the scene investigation has to be done carefully. The day before the live work, the experienced person in charge or staff must be sent to the scene to survey the work environment, understand the complexity and crossover of the work content and so on, to develop a reasonable work plan, and then formulate the corresponding working instruction combined with the specific circumstances of the unit.

\section{Cooperation management of the live work team and the maintenance team}

At present, most live work companies pay attention to the safety management of the live work team and the maintenance team, but there are many management loopholes in the joint overhaul. Sometimes the maintenance team should climb the pole after live work team has taken insulation measures. But sometimes in order to grab the time, the maintenance team climbs the pole to do work before the live work team carries out insulation isolation or it is not fully insulated, and when the operators are working during the power outage, they do less or even do not do insulation protection measures, or hang grounding wire, which is extremely dangerous. This requires the company to restrain staff in the form of system, increase the intensity of implementation, so as to eliminate the above safety risks.

\section{Conclusion}

In the distribution live work, the air and the insulation boom type aerial are the best insulation protection medium for operators and insulated clothing, insulated blankets, insulated sheath are the reserved protection. Due to the complexity of the wires, it is difficult to keep the enough safe distance between the operators and the charged part all the time. In order to ensure the safety of the operators, they are required to wear protective equipment whether it is indirect or direct work. The safe work mode should be adopted by combining main insulation and auxiliary insulation, and taking the multi-layer protection as backup, the tools be used correctly, working procedures are implemented strictly. Only if the safety of persons and equipment are ensured, can the safe and healthy development of the distribution live work be ensured.

\section{References}

[1] HU Yi.Research and development of live working technology on transmission and distribution lines[J].High Voltage Engineering,2006,32(11):1-10.

[2] SHU Yin-biao,HU Yi,LI Xue.Maintenance and live working technology for ultra high voltage transmission line[J].High Voltage Engineering,2007,33(6):1-5. 
[3] HU Jian-xun,LIU Kai,LIU Ting,et a1. An experimental research of living work on high altitude compact $500 \mathrm{kV}$ transmission line[J].High Voltage Apparatus,2010,46(4): 35-39.

[4] ZHANG Jin,JI Sheng-chang,SHEN Qi,et a1.Study on flashover voltage of $35 \mathrm{kV}$ insulator strings hot washed by water vapor[J].High Voltage Apparatus,2010,46(7):61—65.

[5] IEEE Committee Report.Recommendations for safety in live line maintenance[J].IEEE Transactions on Power Apparatus and Systems,1968,87(2):346-352.

[6] State Grid Corporation of China.The power supply enterprise safety risk assessment standard[M].Beijing:China Electric Power Press,2009.

[7] SAATY T L.Fundamentals of the analytic hierarchy process [M]. Pittsburgh: RWS Publications, 2000.

[8] DAGDEVIREN M,YUKSEIJ.Developing a fuzzy analytic hierarchy process(AHP)model for behavior—based safety management[J].Information Sciences,2008,178(6):1717- 1733. 